\title{
MATLAB GUI (graphical user interface) for the design of GRIN components for optical systems as an educational tool
}

A. Gómez-Varela, C. Bao-Varela

A. I. Gómez-Varela, C. Bao-Varela, "MATLAB GUI (graphical user interface) for the design of GRIN components for optical systems as an educational tool," Proc. SPIE 9289, 12th Education and Training in Optics and Photonics Conference, 92891W (17 July 2014); doi: 10.1117/12.2070756

SPIE Event: 12th Education and Training in Optics and Photonics Conference, 2013, Porto, Portugal 


\title{
MATLAB GUI (Graphical User Interface) for the design of GRIN components for optical systems as an educational tool
}

\author{
A. I. Gómez-Varela and C. Bao-Varela* \\ Microoptics and GRIN Optics Group, Facultade de Física and Facultade de Óptica e Optometría, \\ Universidade de Santiago de Compostela, Campus Vida s/n 15782, Santiago de Compostela, Spain
}

\begin{abstract}
New technologies and the available computing tools are becoming more important every day in the teaching evolution. The use of Graphical User Interfaces (GUI) with MATLAB enables the implementation of practical teaching methodologies to make easier the comprehension of a given subject. In this work, we report on the application of GUIs in order to provide the students with a simple tool for a better understanding on how to design GRIN elements for optical systems. Another GUIs advantage is that they can be converted to an executable file, so any student could use the interface in their own computer without having a MATLAB license. We present a graphical interface to show the performance of an optical device for controlling beam size and for deflecting light for coupling purposes, by a simple geometrical optics study, in a tapered GRIN lens illuminated by a parallel beam of tilted rays. We also show a graphical interface to obtain the maximum coupling efficiency between fundamental modes of two single-mode fibers by a scaling operation carried out by a GRIN fiber lens. With this interface the students can vary the magnification and the image plane in order to get the more suitable GRIN fiber lens to maximize the coupling efficiency between two fibers.
\end{abstract}

Keywords: Matlab GUI, active learning, GRIN optics, light deflection, beam size control, GRIN fiber lens

\section{INTRODUCTION}

New technologies are becoming indispensable in all education levels and the development of educational software for promoting active learning is one of the more remarkable aims. Integrating computing applications in conjunction with conventional classroom practices can be a very useful way to improve students' skills. In numerous degrees as, for example, Physics and Engineering degrees, it happens very often that theoretical and practical teaching must complement one another. However, this is not always easy to accomplish given principally to both the lack of time to dedicate to laboratory work and the available resources, since optics experiments use to require from expensive instruments and materials. In this way, the use of applications for simulating practical situations can be an effective manner of supplement the academic training of the students in those cases where the implementation of the experiment is not possible and also to complement the theoretical lessons. In this regard, several resources have been developed for the teaching of optics and other science and engineering disciplines ${ }^{1-4}$.

In optics communications very often arises the necessity to joint together two single-mode fibers or light sources to single-mode fibers, operation that can be carry out by GRIN (GRadient-INdex) lenses ${ }^{5}$. In this way, two common uses of GRIN lenses are for controlling beam size and deflecting light in a tapered GRIN lens, when illuminated by a parallel beam of tilted rays, as well as for obtaining the maximum coupling efficiency between fundamental modes of two singlemode fibers, using in this case a GRIN fiber lens. GRIN lenses consist of a cylinder of inhomogeneous dielectric material where the refractive index distribution has a maximum at the cylinder axis and decreases continuously from the axis to the edges along the transverse directions. In such a components, ray light describes sinusoidal paths because of the variation of the refractive index. In this work we present two graphical user interfaces created to simulate these two

*carmen.bao@usc.es; phone +34881813168;

12th Education and Training in Optics and Photonics Conference, edited by

Manuel F. P. C. Martins Costa, Mourad Zghal, Proc. of SPIE Vol. 9289, 92891W

(c) 2014 SPIE, OSA, IEEE, ICO · doi: 10.1117/12.2070756

Proc. of SPIE Vol. $928992891 \mathrm{~W}-1$ 
possible applications of GRIN lenses in optical connections, that is, single-mode coupling by tapered and non-tapered GRIN fiber lenses. The use of the interfaces allows students to focus on the concepts of the operation avoiding the complicated mathematical formulation that lies behind both applications. The educational software proposed in this paper has been designed using the MATLAB GUIDE environment ${ }^{6}$, a powerful tool that permits to obtain user-friendly and versatile Graphical User Interfaces (GUIs).

\section{GRIN LENSES FOR OPTICAL CONNECTIONS: THEORETICAL BACKGROUND}

GRadient INdex (GRIN) optics is the branch of Optics covering optical effects produced by a gradual and smooth variation of the refractive index of a material with position. Some optical systems for optics communications can be designed using GRIN lenses. This kind of lenses can perform typical functions as on-axis and off-axis imaging, collimation and focusing in optical systems ${ }^{7-10}$. These systems are based on imaging and transforming properties of such a media in order to carry out specific functions. In this work we center in two typical configurations that can be performed with GRIN lenses, that is, beam-size control and light deflection and single-mode fibers coupling.

\subsection{GRIN light deflector and beam-size controller}

In this section we show the performance of an optical device for controlling beam size and for deflecting light in coupling purposes by a simple geometrical optics study in a tapered GRIN lens, illuminated by a parallel beam of tilted rays $^{7}$.

Let us consider a GRIN media of thickness $d$ and semi-aperture a, with refractive index profile given by the following expression

$$
\mathrm{n}^{2}(\mathrm{r}, \mathrm{z})=\mathrm{n}_{0}^{2}\left[1-\mathrm{g}^{2}(\mathrm{z}) \mathrm{r}^{2}\right] ; \quad \mathrm{r}=\left(\mathrm{x}^{2}+\mathrm{y}^{2}\right)^{1 / 2} \leq \mathrm{a} ; \quad 0 \leq \mathrm{z} \leq \mathrm{d}
$$

with $\mathrm{n}_{0}$ being the refractive index at the lens axis and $\mathrm{g}(\mathrm{z})$ the gradient parameter.

Light propagation in GRIN media can be represented by a ray describing a path of light energy. The ray trajectory can be obtained from a second-order differential equation, whose general solution can be represented by the position $\mathrm{r}$ and the slope $\dot{r}$ of the ray at any given $z$ as ${ }^{8}$

$$
\left.\left.\stackrel{(\cdot)}{\mathrm{r}}(\mathrm{z})=\mathrm{r}_{0} \stackrel{(\cdot)}{\mathrm{H}}_{\mathrm{f}} \mathrm{z}\right)+\dot{\mathrm{r}}_{0} \stackrel{(\cdot)}{\mathrm{H}}_{\mathrm{a}} \mathrm{z}\right)
$$

Eq. (2) is a linear combination of two independent particular solutions: the axial, $\mathrm{H}_{\mathrm{a}}$, and the field, $\mathrm{H}_{\mathrm{f}}$, rays. In this expression, the coefficients $r_{0}$ and $\dot{r}_{0}$ are the position and slope of the ray at the input plane $z=0$. On the other hand, the linear algebraic nature of Eq. (2) allows us to express the paraxial propagation of light in GRIN materials by means of the ray-transfer matrix or ABCD matrix. This matrix relates the position and slope of any meridional rays at to axial values as follows

$$
\left(\begin{array}{c}
r(z) \\
\dot{r}(z)
\end{array}\right)=\left(\begin{array}{cc}
H_{f}(z) & H_{a}(z) \\
\dot{H}_{f}(z) & \dot{H}_{a}(z)
\end{array}\right)\left(\begin{array}{c}
r_{0} \\
\dot{r}_{0}
\end{array}\right)
$$

When a GRIN lens is illuminated by a parallel beam of rays with slope $\dot{r}_{0}=\theta_{0} / n_{0}$, where $\theta_{0}$ is the deflection angle of the input beams, not all the impinging rays on the lens will be confined through it. For the imaging condition

$$
\mathrm{H}_{\mathrm{a}}\left(\mathrm{z}_{\mathrm{m}}\right)=\dot{\mathrm{H}}_{\mathrm{f}}\left(\mathrm{z}_{\mathrm{m}}\right)=0
$$

the position and slope of the marginal rays at a given length $\mathrm{z}_{\mathrm{m}}$ (where $\mathrm{m}$ is an integer) are proportional. In other words, input rays produce a parallel output beam of rays in other direction whose scaling factor for position (transverse magnification) and for slope (angular magnification) are given by ${ }^{9}$ 


$$
\begin{aligned}
& \mathrm{M}_{\mathrm{t}}=\mathrm{H}_{\mathrm{f}}\left(\mathrm{z}_{\mathrm{m}}\right)=(-1)^{\mathrm{m}}\left(\frac{\mathrm{g}_{0}}{\mathrm{~g}\left(\mathrm{z}_{\mathrm{m}}\right)}\right)^{1 / 2} \\
& \mathrm{M}_{\mathrm{a}}=\dot{\mathrm{H}}_{\mathrm{a}}\left(\mathrm{z}_{\mathrm{m}}\right)=(-1)^{\mathrm{m}}\left(\frac{\mathrm{g}\left(\mathrm{z}_{\mathrm{m}}\right)}{\mathrm{g}_{0}}\right)^{1 / 2}
\end{aligned}
$$

where $\mathrm{g}_{0}$ is the value of the gradient parameter at $\mathrm{z}=0$. In this way, it is possible to obtain an optical element that can change light propagation and control the beam size of the incident light beam by simply cutting the tapered GRIN lens at the $\mathrm{z}_{\mathrm{m}}$ lengths.

We apply now these results to a tapered GRIN lens whose taper function is given by

$$
\mathrm{g}(\mathrm{z})=\frac{\mathrm{g}_{0}}{1-\left(\frac{\mathrm{z}}{\mathrm{L}}\right)^{2}}
$$

The equi-index surfaces for the refractive index are revolution paraboloids around the $\mathrm{z}$-axis with $\mathrm{L}$ being the distance from $z=0$ to the common apex of the equi-index lines. In this case, the $z_{m}$ lengths that fulfills the imaging condition are given by

$$
\mathrm{z}_{\mathrm{m}}=\mathrm{L} \tanh \left(\frac{\mathrm{m} \pi}{\mathrm{g}_{0} \mathrm{~L}}\right)
$$

\subsection{GRIN fiber lens}

A GRIN fiber lens (GFL) is an optical component manufactured with a multimode GRIN fiber of small diameter and strong focusing fused to single mode fibers (SMF) and aligned by surface tension for connection purposes between fundamental mode fields of different size. In this way, GFLs can couple elliptical modal fields in an optimal way ${ }^{6}$. GFLs present some advantages over other components as small size, low cost, easy production and alignment with single-mode fibers, common fiber/GFL diameter, and stable (fused) fiber-GFL interface ${ }^{11}$. In Figure 1 an optical connector designed using a GFL fused to monomode fibers is shown.

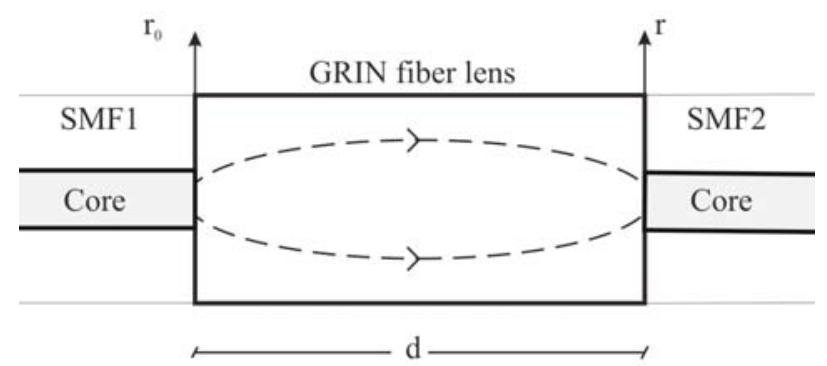

Figure 1. Schematic drawing of the operation of the GRIN lens optical connector fused to the end of two monomode fibers.

We consider now that the refractive index profile of the GFL of thickness $d$ is represented by Eq. (1) and that the integral transformation performed by the lens can be written as

$$
\psi(\mathrm{x}, \mathrm{y} ; \mathrm{d})=\int_{\mathrm{R}^{2}} \mathrm{~K}\left(\mathrm{x}_{0}, \mathrm{y}_{0}, \mathrm{x}, \mathrm{y} ; \mathrm{d}\right) \psi_{1}\left(\mathrm{x}_{0}, \mathrm{y}_{0}\right) \mathrm{dx_{0 }} d \mathrm{y}_{0}
$$

where K represents the Point Spread Function and $\psi_{1}$ is the mode field of the first single mode fiber (SMF1). 
If $\Psi_{1}$ is the fundamental mode at the output face of the SMF1, a replica of $\Psi_{1}$ on the input face of the second fiber, SMF2, can be obtained when the thickness of the GFL is such that the imaging condition at its output face, given by Eq. (4), is fulfilled. At this condition, an exact reconstruction of the mode field $\Psi_{1}$ is achieved with a scaling factor given by Eq. (5).

After a straightforward calculation ${ }^{7}$, the maximum coupling efficiency for the GFL connector is given by

$$
\eta_{\max }=\frac{4 g_{0}}{g\left(z_{m}\right)\left[\frac{w_{x 2}}{w_{x 1}}+\frac{w_{y 2}}{w_{y 1}}\right]^{2}}
$$

In Eq. (10), $\mathrm{w}_{\mathrm{x} 1}, \mathrm{w}_{\mathrm{x} 2}, \mathrm{w}_{\mathrm{y} 1}$ and $\mathrm{w}_{\mathrm{y} 2}$ represent the beam half-widths of the normalized Gaussian mode field along the $\mathrm{x}$ and y axes.

\section{GRIN LIGHT DEFLECTOR AND BEAM-SIZE CONTROLLER AND GRIN FIBER LENS GRAPHICAL USER INTERFACES}

We present here two graphical user interfaces created to explain the two applications of GRIN lenses in optical communications described above. Both GUIs have been designed using the MATLAB GUIDE environment, which allows us to perform tasks interactively through the GUIDE Layout Editor in an easy way.

In Figure 2 we can see the main window of the graphical user interface designed to show the performance of an optical device for controlling beam size and deflecting light (with coupling purposes) in a tapered GRIN lens illuminated by a parallel beam of tilted rays. The taper function of this lens is given by Eq. (7). The user can preview how the variation of the semi-aperture and the deflection angle of the input beams influence on ray light propagation in the GRIN lens. When the user chose the values of these parameters, two figures are displayed on the interface: at the top, the ray tracing for a GRIN lens cut at $z_{1}$ (for which the first zero of $H_{a}$ is obtained) is shown; below, the same device is represented but now the lens has been cut at a length $z_{2}$ in order to illustrate the behaviour of ray lights in thicker elements. We can see that depending on the value of a and $\theta_{0}$, and on the $z_{\mathrm{m}}$ length of the tapered GRIN lens, different output deflection angles and also different output beam sizes are obtained. It is interesting to highlight that, for the principle of reversibility of the path of ray lights, the device can also be employed as a beam expander and for beam slope reduction. The ray tracing has been made for input and GRIN lens parameters: $\mathrm{a}=1 \mathrm{~mm}, \mathrm{~g}_{0}=0.2325 \mathrm{~mm}^{-1}, \mathrm{n}_{0}=1.604, \mathrm{~L}=60 \mathrm{~mm}$ and $\theta_{0}=5^{\circ}$.

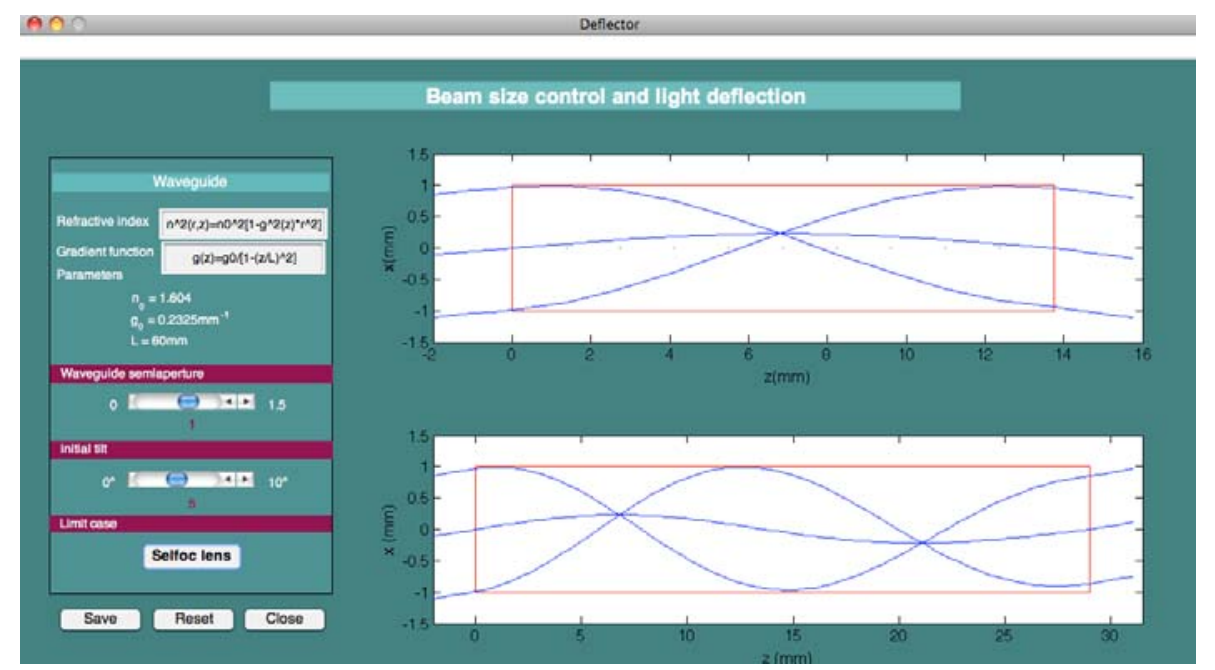

Figure 2. Main window of the graphical user interface designed for the study of light deflector and beam size controller with a tapered GRIN lens cut at $\mathrm{z}_{1}$ (figure at the top) and cut at $\mathrm{z}_{2}$ (figure at the bottom). 
As we pointed out in the previous section, maximum coupling efficiency between fundamental modes of two singlemode fibers can be obtained by a scaling operation carried out by a GRIN fiber lens. In Figure 3 and Figure 4 we can see the layout of an application for analysing monomode fiber coupling by GRIN lenses. In this GUI application the users can choose between two gradient parameters of the GRIN lens, that is, diverging linear (Figure 3) and converging parabolic (Figure 4). For each gradient parameter it is also possible to vary the value of the magnification and image plane. Once these parameters are selected by the user, three graphics are displayed to the right on the application window, where users can see how the beam waist evolves inside the GRIN lens (top) and study the coupling efficiency (center) and the coupling losses (bottom) depending on the GRIN lens thickness. When comparing the three plots in Figure 3, it results very intuitive to figure out that the maximum coupling efficiency is obtained for those lengths of the GFL where the image condition is fulfilled and also that it diminishes with the lens length. Besides, the user can observe that the contrary occurs for the coupling losses and that the GFL thickness required for maximum coupling efficiency varies depending on the gradient index parameter profile, magnification and image plane. In the case of the converging parabolic gradient parameter in Figure 4, it is interesting to note that the contrary happens for the maximum coupling efficiency and the coupling losses, given that the first one increases with the thickness of the coupling GRIN lens and the second one diminishes. In Figure 3, the ray tracing has been made for input, single mode fibers and GRIN lens parameters collected in Table 1. For Figure 4, the parameter values for simulations are shown in Table 2. In both cases, $\lambda=1300 \mathrm{~nm}$.

Table 1. Parameter values for the coupling of two single-mode fibers by GFL simulated in Figure 3.

\begin{tabular}{|c|c|c|}
\hline SMF1 & GRIN lens & SMF2 \\
\hline Core $=6 \mu \mathrm{m}$ & $\mathrm{g}(\mathrm{z})=\mathrm{g}_{0} /(1+\mathrm{z} / \mathrm{L})$ & Core $=9 \mu \mathrm{m}$ \\
\hline $\mathrm{W}_{\mathrm{x} 1}=2.5 \mu \mathrm{m}$ & $\mathrm{g}_{0}=6 \mathrm{~mm}^{-1}$ & $\mathrm{w}_{\mathrm{x} 2}=2.75 \mu \mathrm{m}$ \\
\hline $\mathrm{w}_{\mathrm{y} 1}=2.75 \mu \mathrm{m}$ & $\mathrm{n}_{0}=1.5916$ & $\mathrm{w}_{\mathrm{y} 2}=3.05 \mu \mathrm{m}$ \\
\hline
\end{tabular}

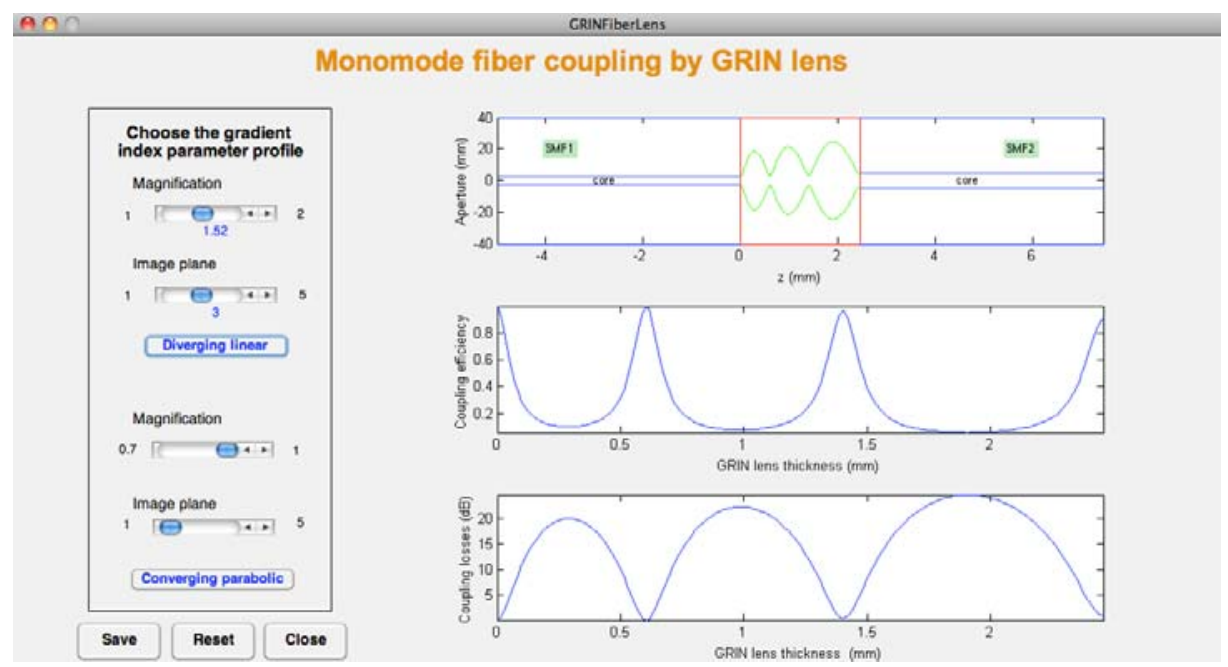

Figure 3. Graphical user interface front-end for simulation of a GRIN fiber lens with a diverging linear gradient parameter profile operation. 
Table 2. Parameter values for the coupling of two single-mode fibers by GFL simulated in Figure 4.

\begin{tabular}{|c|c|c|}
\hline SMF1 & GRIN lens & SMF2 \\
\hline Core $=9 \mu \mathrm{m}$ & $\mathrm{g}(\mathrm{z})=\mathrm{g}_{0} / 1-(\mathrm{z} / \mathrm{L})^{2}$ & Core $=6 \mu \mathrm{m}$ \\
\hline $\mathrm{W}_{\mathrm{x} 1}=2.75 \mu \mathrm{m}$ & $\mathrm{g}_{0}=6 \mathrm{~mm}^{-1}$ & $\mathrm{w}_{\mathrm{x} 2}=2.5 \mu \mathrm{m}$ \\
\hline $\mathrm{w}_{\mathrm{y} 1}=3.25 \mu \mathrm{m}$ & $\mathrm{n}_{0}=1.5916$ & $\mathrm{w}_{\mathrm{y} 2}=2.75 \mu \mathrm{m}$ \\
\hline
\end{tabular}

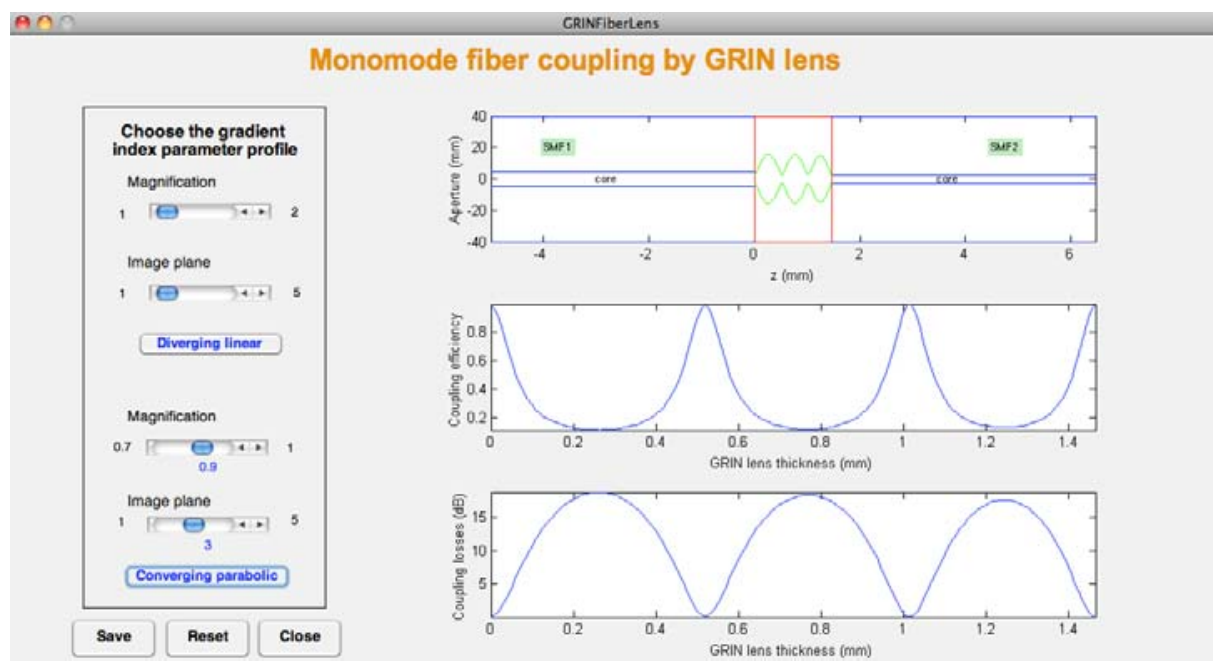

Figure 4. Graphical user interface front-end for simulation of a GRIN fiber lens with a converging parabolic gradient parameter profile operation.

\section{CONCLUSIONS}

In this work we present two graphical user interfaces, designed using the MATLAB GUIDE environment, for the teaching of some applications of GRIN lenses in optics communications. In particular, the graphical user interfaces simulate two optical configurations designed for beam-size control and light deflection by tapered GRIN lenses and for obtaining the maximum coupling efficiency possible between fundamental modes of two single-mode fibers, by a scaling operation, performed by a GRIN fiber lens. The GUIs allow the students to understand the basis of those applications of GRIN lenses without the necessity of going into details in the mathematical model. In this way, the interaction of the student with the GUI is very simple and intuitive and makes possible for the student to analyse qualitatively both optical configurations. The reported GUIs are intended to perform a complementary role in Science and Engineering education as part of a "virtual lab", in order to supplement theoretical and practical sessions and for reinforcing the knowledge acquired by the students.

\section{ACKNOWLEDGEMENTS}

This work has been supported by the Ministerio de Ciencia e Innovación (MAT2010-18519). Besides, Ana I. GómezVarela wants to acknowledge the financial support from the FPU (Formación de Profesorado Universitario) grant (Ministerio de Educación, Cultura y Deporte, Spain). 


\section{REFERENCES}

[1] Frances, J., M. Perez-Molina, Bleda, S., Fernandez, E., Neipp, C. and Belendez, A., "Educational Software for Interference and Optical Diffraction Analysis in Fresnel and Fraunhofer Regions Based on MATLAB GUIs and the FDTD Method", IEEE Transactions on Education, 55(1), 118-125 (2012).

[2] http://www.uv.es/indoptic/materialArticulos.html.

[3] http://grupoorion.unex.es/optoelectronicaweb/index.html.

[4] Ali H. Assi, Maitha H. Al Shamisi and Hassan A. N. Hejase, "MATLAB GUI Application for Teaching Electronics, Engineering Education and Research Using MATLAB”, Dr. Ali Assi (Ed.), ISBN: 978-953-307656-0, InTech (2011).

[5] Gilsdorf, R. W., and Palais, J. C., "Single-mode fiber coupling efficiency with graded-index rod lenses", Appl. Opt., 33(16), 3440-3445 (1994).

[6] Marchand, P. and Holland, O. T., [Graphics and GUIs with Matlab], Chapman \& Hall/CRC (2002).

[7] Gomez-Reino, C., Perez, M. V., and Bao, C., [Gradient-Index Optics: Fundamentals and Applications], Springer, Berlin, Chap. 9 (2010).

[8] Luneburg, R. K., [Mathematical Theory of Optics], University of California Press, Berkeley (1964).

[9] Gómez-Reino, C, Pérez M.V., Bao C., Flores-Arias M.T., "Design of GRIN optical components for coupling and interconnects" Laser Photonics Rev. 2, 203-215 (2008).

[10] Shealy, D.L., Chao, S.-H., "Design of GRIN laser beam shaping system", Proc. SPIE 5525, Laser Beam Shaping V, 138, 138-147 (2004).

[11] Lizuka, K., [Engineering Optics], Springer-Verlag, Berlin, Chap. 13 (1987). 